\title{
Thermal tuning of mechanically induced long-period fiber grating
}

\author{
Takashi Yokouchi, Yoshifumi Suzaki, Kiyoshi Nakagawa, Makoto Yamauchi, \\ Masanori Kimura, Yasuo Mizutani, Susumu Kimura, and Seiki Ejima
}

\begin{abstract}
We have developed a wideband tunable optical filter that uses a long-period fiber grating (LPFG) in which both resonance wavelength and its signal attenuation can be adjusted. We create the grating mechanically by pressing a spring coil to an optical fiber. We achieve continuous fine tuning of wavelength and attenuation by varying the temperature of the LPFG. The adjustable ranges of the LPFG are more than $200 \mathrm{~nm}$ in resonance wavelength and more than $10 \mathrm{~dB}$ in signal attenuation. () 2005 Optical Society of America
\end{abstract}

OCIS codes: $\quad 050.2770,060.2310,060.2340,120.2440$.

\section{Introduction}

A long-period fiber grating (LPFG) is a loss filter that uses mode coupling between the fundamental guided mode and forward-propagating cladding modes in an optical fiber. ${ }^{1}$ Owing to its simple structure and excellent characteristics, the device is used for many applications, such as amplitude equalizing devices for optical amplifiers and various types of optical fiber sensor.

The LPFG has been conventionally formed in an optical fiber by inducing periodic changes in the fiber's refractive index with period lengths of typically several hundred micrometers. The index variation was achieved by irradiation with UV light through an amplitude mask. ${ }^{1}$ The method of periodic local heating of the optical fiber by $\mathrm{CO}_{2}$ laser irradiation was also developed.2,3 These fabrication methods give permanently fixed LPFG parameters, such as grating period, modulation amplitude, and total length. Recently, in a search for flexibility in applications, interest has shifted to tunable LPFGs. For this purpose, a mechanical method of inducing refractiveindex changes along the fiber was also proposed. Be-

T. Yokouchi, Y. Suzaki, K. Nakagawa, and S. Ejima (ejima@eng. kagawa-u.ac.jp) are with the Faculty of Engineering, Kagawa University, 2217-20 Hayashi-cho, Takamatsu, Kagawa, 761-0396 Japan. M. Yamauchi, M. Kimura, Y. Mizutani, and S. Kimura are with the Shinko Electric Wire Company, Ltd., 1298-12 Shido, Shido-cho, Sanuki, Kagawa, 769-2101 Japan.

Received 16 December 2004; revised manuscript received 3 March 2005; accepted 25 March 2005.

0003-6935/05/245024-05\$15.00/0

(C) 2005 Optical Society of America cause the period is as large as several hundred micrometers, one can induce the index modulation mechanically, for example, by pressing the fiber between a periodically grooved plate and a flat plate ${ }^{4}$ or by winding a nylon string around the fiber periodically. ${ }^{5}$

Besides these, other methods were also reported, for example, tuning resonance wavelength by periodically spaced electrical heating of the metal film coated on the LPFG, ${ }^{6}$ adjusting signal attenuation by temperature control of a periodically corrugated substrate, which induces thermal strains on the LPFG, ${ }^{7}$ and controlling the spectral shape by adjusting the temperature of the LPFG section, using divided coil heaters. ${ }^{8}$ Fine tuning of resonance wavelength by bending the LPFG with a piezoelectric actuator was reported, ${ }^{9}$ but high voltage and fine adjustment are needed there. Each method has its strengths and weaknesses in actual applications.

A tunable optical filter requires control of the resonance wavelength and signal attenuation independently. The resonance wavelength should be adjustable without affecting the signal attenuation, and the signal attenuation should be adjustable without affecting the resonance wavelength. In search of such a tunable LPFG device, we developed a method of fabricating a mechanically tunable LPFG by using an impressing spring..$^{10}$ Recently, by adding temperature control to this mechanical LPFG, we made it tunable in a range of $200 \mathrm{~nm}$ in wavelength and adjustable in signal attenuation by more than $10 \mathrm{~dB}$. We describe the details of this tunable LPFG in the following sections. 


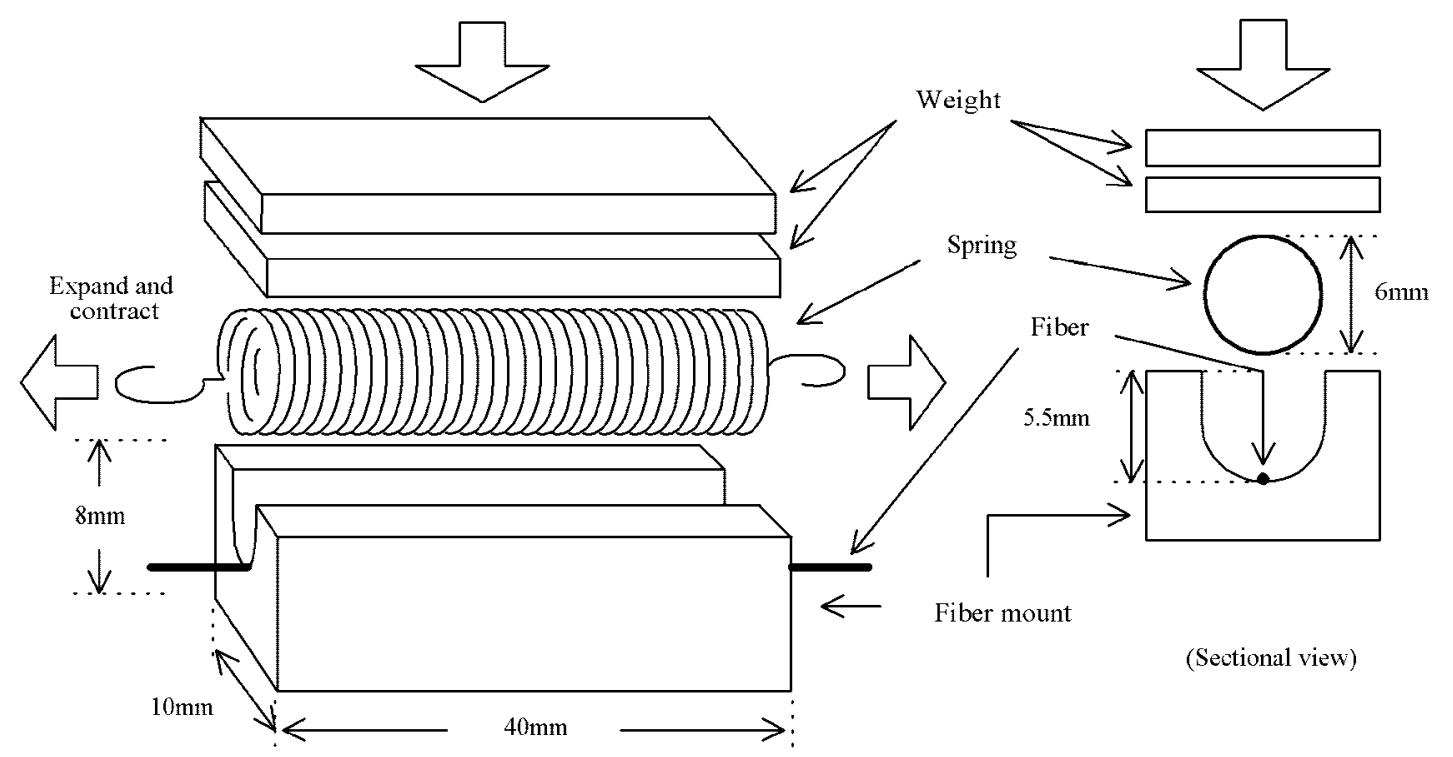

Fig. 1. Setup for mechanically tuning a LPFG with a coiled spring.

\section{Mechanically Induced Tunable Long-Period Fiber Grating}

In a LPFG, the fundamental mode of incident light couples with cladding modes. The cladding mode is lost to outside the fiber; thus the amplitude of the fundamental mode is decreased. Resonance wavelength $\lambda_{m}$ of the LPFG is given by

$$
\lambda_{m}=\Lambda\left(n_{\text {core }}-n_{\text {clad }}^{m}\right),
$$

where $\Lambda$ is the grating period of the LPFG, $n_{\text {core }}$ is the refractive index of the $\mathrm{LP}_{01}$ fundamental mode, and $n_{\text {clad }}{ }^{m}$ is the index of $\operatorname{LP}_{0 m}(m=2,3,4 \ldots)$ cladding modes. Resonance wavelength $\lambda_{m}$ can be adjusted by proper selection of grating period $\Lambda$.

The setup for mechanically tuning the LPFG by using a coiled spring is shown in Fig. 1. We can adjust grating period $\Lambda$ by expanding the length of the coiled spring. An optical fiber is placed on the bottom of the fiber mount, i.e., the U-shaped metal base. The spring is inserted there, and a weight is placed on the spring to press it against the fiber. The spring is made from stainless steel with a diameter of $0.5 \mathrm{~mm}$, a coil diameter of $6 \mathrm{~mm}$, and a coil section length of $40 \mathrm{~mm}$. The length of the LPFG is the same as that of U-shaped base. The optical fiber that we used is standard single-mode fiber (SMF-28, Corning, Inc.). Wideband optical light was injected into the fiber from an amplified spontaneous emission light source (Fiber Lab., ASE-FL7701), and the light that passed through the LPFG was measured by an optical spectrum analyzer (Ando, AQ6317C). The transmission spectra in the wavelength range $1400-1650 \mathrm{~nm}$ are shown in Fig. 2 for two coil pitches of the spring, 0.6 and $0.625 \mathrm{~mm}$. As shown in the figure, three attenuation bands appeared in this wavelength range. They are apparently the same as those of mechanical LPFGs reported in Refs. 4 and 5. Here we call them the first, the second, and the third attenuation bands from the short-wavelength side.

Figure 3 shows how the resonant wavelengths of the first, second, and third attenuation bands are proportional to the coil pitch of the spring, that is, to the grating period $\Lambda$. Figure 4 shows transmission

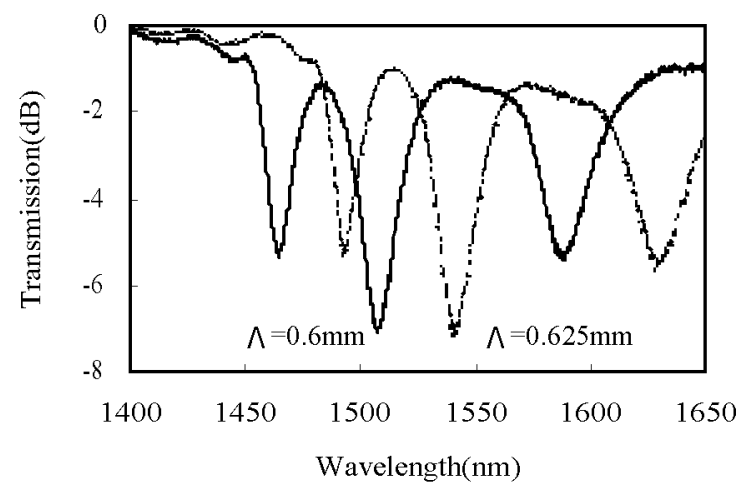

Fig. 2. Measured transmission spectra of LPFGs with grating periods $\Lambda=0.6$ and $0.625 \mathrm{~mm}$.

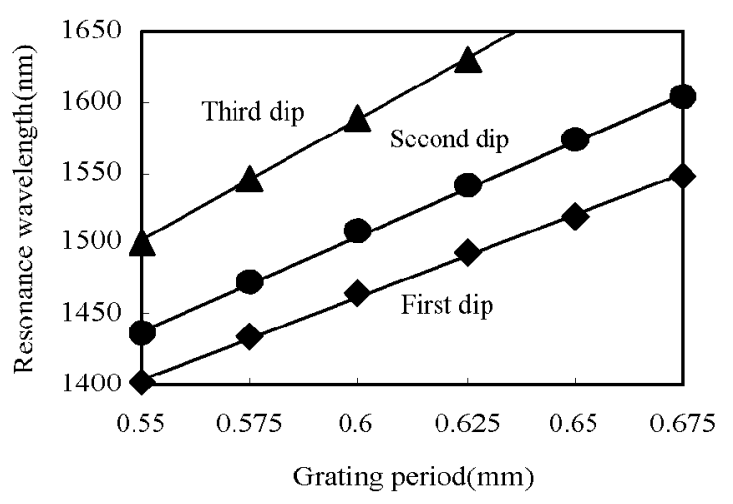

Fig. 3. Resonance wavelength for several grating periods. 


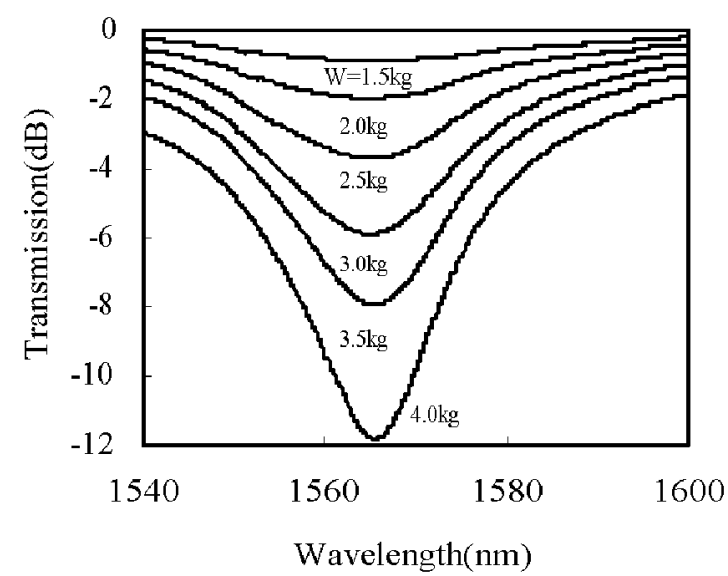

Fig. 4. Measured transmission spectra of LPFG with $\Lambda$ $=0.593 \mathrm{~mm}$ and several weights from 1.5 to $4.0 \mathrm{~kg}$.

spectra of the LPFG with grating period $\Lambda$ fixed at $0.593 \mathrm{~mm}$ and with several impressing weights from 1.5 to $4.0 \mathrm{~kg}$ on the fiber. The measured spectra are for the third attenuation band. The amount of attenuation increases as the impressing weight is increased. It should be noted that the central value of the resonance wavelength does not change for different weights.

\section{Thermal Control of Resonance Wavelength}

We studied, first, the dependence of the resonance wavelength on the ambient temperature by placing the device shown in Fig. 1 into a thermostatic oven. Coil pitch, that is, grating period $\Lambda$, was set to $0.58 \mathrm{~mm}$. The light from the amplified spontaneous emission light source was passed through the LPFG, and the transmission spectra were monitored in situ by the optical spectrum analyzer. The LPFG device's temperature in the thermostatic oven was changed in increments of $20^{\circ} \mathrm{C}$ from $0{ }^{\circ} \mathrm{C}$ to $+100{ }^{\circ} \mathrm{C}$, and the evolution of the third attenuation band was measured. The temperature dependence of the LPFG resonance wavelength is shown in Fig. 5. The amount of the shift of the resonance wavelength is $6 \mathrm{~nm}$ for the temperature change from $0{ }^{\circ} \mathrm{C}$ to $+100{ }^{\circ} \mathrm{C}$, giving $0.060 \mathrm{~nm} /{ }^{\circ} \mathrm{C}$, which is close to the amounts of shift reported previously. ${ }^{1,11,12,13}$ Our results show that we

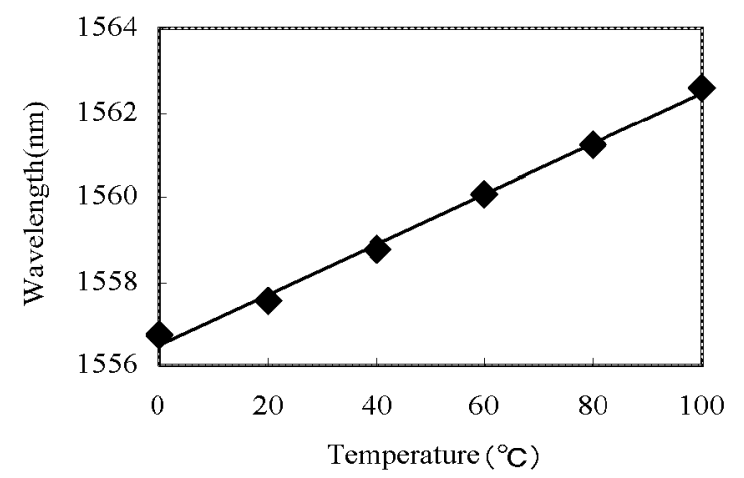

Fig. 5. Temperature dependence of LPFG with $\Lambda=0.58 \mathrm{~mm}$.

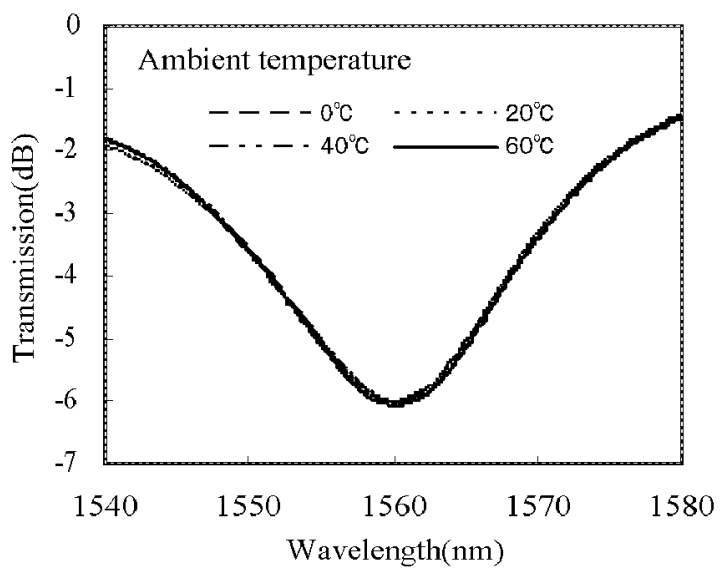

Fig. 6. Measured transmission spectra of LPFG for several ambient temperatures with the fiber mount temperature fixed at $60{ }^{\circ} \mathrm{C}$.

can fine tune the wavelength as much as $6 \mathrm{~nm}$ by temperature control of the LPFG, making it continuously tunable over $200 \mathrm{~nm}$. Next we studied the effects of ambient temperature on the LPFG maintained at a constant temperature by local heating. We heated the LPFG to a given fixed temperature, changed the ambient temperature, and measured the spectra to see whether the resonance wavelength and signal attenuation remained the same. The LPFG of Fig. 1 was placed on a small hot plate, and the hot plate was put into a thermostatic oven. A temperature sensor was attached to the fiber mount of the LPFG for temperature monitoring. The grating pe$\operatorname{riod} \Lambda$ was $0.58 \mathrm{~mm}$, the same as in the experiment described above.

The temperature in the thermostatic oven was changed in increments of $20^{\circ} \mathrm{C}$ from $0{ }^{\circ} \mathrm{C}$ to $+60{ }^{\circ} \mathrm{C}$, and the voltage supplied to the heater was adjusted such that the temperature of the LPFG might be fixed to $60{ }^{\circ} \mathrm{C}$ regardless of any ambient temperature. The transmission spectra are shown in Fig. 6 for ambient temperatures. The resonant wavelengths were $1560.16,1560.16,1560.24$, and $1560.08 \mathrm{~nm}$ at $0{ }^{\circ} \mathrm{C}$, $20^{\circ} \mathrm{C}, 40{ }^{\circ} \mathrm{C}$, and $60{ }^{\circ} \mathrm{C}$, respectively. The shapes of all these spectra were nearly identical. This result indicates that it is possible to control the temperature of the LPFG section locally by use of an external heating source independently of the ambient temperature.

\section{Mechanically Induced Tunable Long-Period Fiber Grating with Thermal Control}

To change the resonance wavelength of the LPFG mechanically by adjusting the spring, first we release the weight pressing on the optical fiber and then we readjust grating period $\Lambda$ by changing the coil spacing of the spring. The weight is then reapplied. This process gives discrete values of the resonance wavelengths. To ensure continuous rather than discrete resonance wavelengths, we added the thermal control described in Section 3. It is possible to do the tuning thermally over $6 \mathrm{~nm}$ when the temperature of 


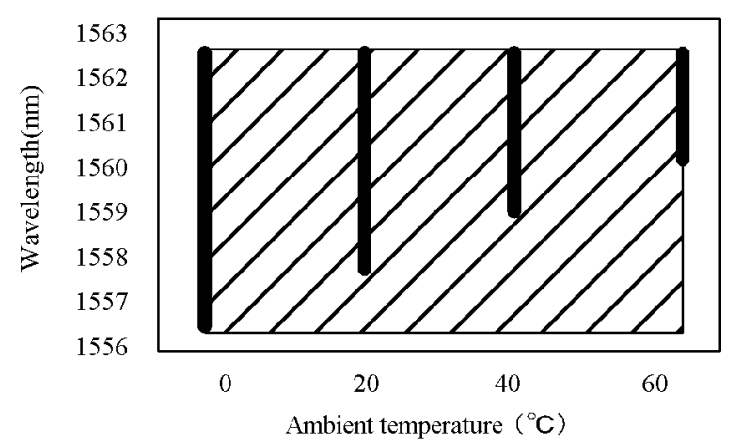

Fig. 7. Range of resonance wavelengths for several ambient temperatures.

the fiber mount is changed from 0 to $100{ }^{\circ} \mathrm{C}$. The LPFG that uses the spring can control the tuning mechanically for more than $200 \mathrm{~nm}$ as described above, and its accuracy in setting the wavelength is $1 \mathrm{~nm}$ or greater. Hence the combination of the mechanical tuning with thermal tuning will give fine continuous tuning for more than $200 \mathrm{~nm}$.

As shown in Fig. 5, when grating period $\Lambda$ is $0.58 \mathrm{~mm}$, we can control the resonance wavelength from 1556.5 to $1562.5 \mathrm{~nm}$ by changing the temperature of the fiber mount from 0 to $100{ }^{\circ} \mathrm{C}$. For the range of ambient temperature from 0 to $60{ }^{\circ} \mathrm{C}$, the range of tunable control of the resonance wavelength is shown in Fig. 7 by the hatched area. To confirm this range of resonance wavelengths, we changed the temperature of the fiber mount to as much as $100{ }^{\circ} \mathrm{C}$ from thermostatic oven temperatures of initially $0,20^{\circ}$, $40^{\circ}$, and $60^{\circ} \mathrm{C}$ and monitored ways in which the wavelength might vary. The results are shown by the thick lines in Fig. 7. For the oven temperature of $+60{ }^{\circ} \mathrm{C}$ the wavelength has been controlled from 1560 to $1562.5 \mathrm{~nm}$, exactly as expected. Here the temperature of the fiber mount was increased from ambient temperature by heating. If a Peltier semiconductor cooling element is used, the controllable range should also be easily extendable to the opposite direction, i.e., a colder temperature covering the entire range from 0 to $100{ }^{\circ} \mathrm{C}$.

Figure 8 shows measured transmission spectra with grating period $\Lambda=0.58 \mathrm{~mm}$ with the weight on

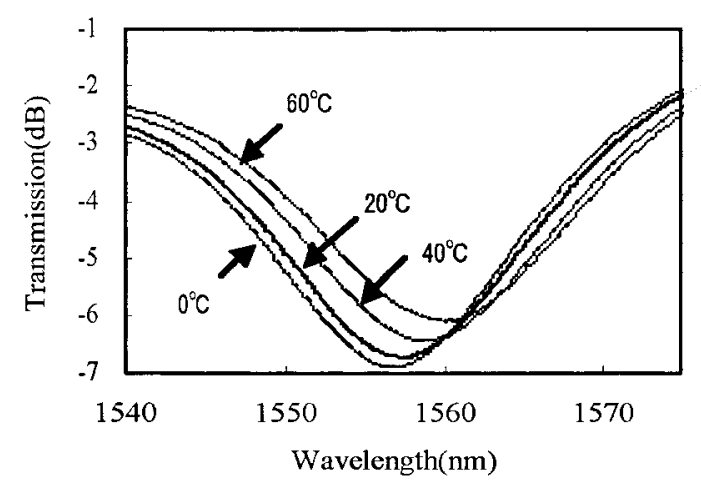

Fig. 8. Measured transmission spectra for four ambient temperatures with a fixed weight.

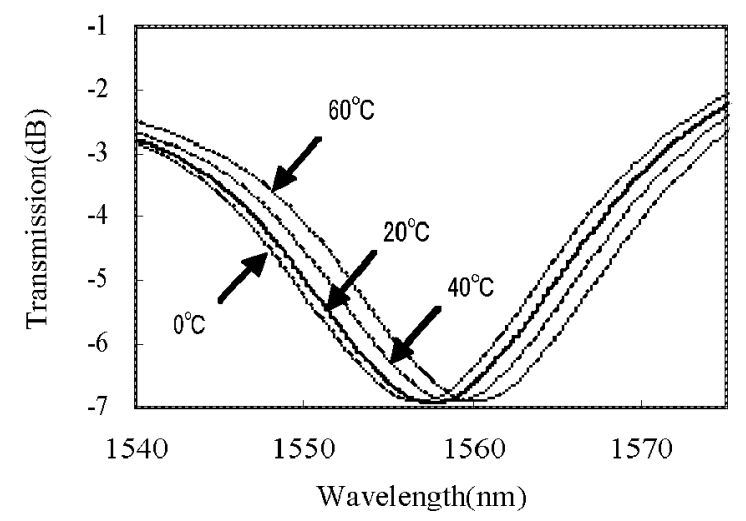

Fig. 9. Measured transmission spectra for four ambient temperatures with adjustable weights.

the fiber fixed at $3.2 \mathrm{~kg}$ and at ambient temperatures of $0,20^{\circ}, 40^{\circ}$, and $60^{\circ} \mathrm{C}$. As the temperature is changed, not only the resonance wavelength but also the amount of attenuation has changed. The amount by which the attenuation has decreased as the temperature is raised is shown in the figure. The temperature dependence of resonance wavelength and attenuation varies with experimental conditions, as reported in Refs. 7, 8, and 14. The variations are attributed to the differences in fiber doping during fiber fabrication and preparation and to the method by which the LPFG is made. It is important to control the amount of attenuation without affecting the resonance wavelength.

Figure 9 shows the results after the weights were adjusted to have the same amount of attenuation for different temperatures: The weights added to that at $0{ }^{\circ} \mathrm{C}$ are $+40,+90$, and $+160 \mathrm{~g}$ at $20^{\circ} \mathrm{C}, 40^{\circ} \mathrm{C}$, and $60^{\circ} \mathrm{C}$, respectively. These values of weights are in agreement with the relation that the amount of attenuation is a quadratic function of weight. ${ }^{10}$ If the dependence of the amount of attenuation on temperature is already known, the pressure on the fiber can be programmed and applied automatically.

Our LPFG has an inherently asymmetric structure, which can be sensitive to polarization. This sensitivity may affect some applications of the device. We intend to investigate these potential effects.

\section{Discussion and Summary}

It has been demonstrated that the tunable longperiod fiber gratings can control both resonance wavelength and signal attenuation. The unique feature of this LPFG is that it is formed only by the force applied to an optical fiber by a spring, unlike the conventional LPFG, for example, which is fabricated by exposure to a UV laser. The spring method has two major advantages: One is that the resonance wavelength can easily be selected and readjusted by the spring and the other is that the signal attenuation level can be adjusted by a change in the pressure of the optical fiber. In the conventional LPFG, the modulation period and the modulation amplitude of the grating are both permanently inscribed in the fiber, 
so it is difficult to change both the resonance wavelength and the signal attenuation level. Furthermore, our LPFG has both an extremely wide tunable range of more than $200 \mathrm{~nm}$ in the resonance wavelength and the capability of continuous tuning over that wavelength range. These ranges were achieved by a combination of mechanical tuning and thermal tuning.

In an actual implementation of this LPFG in various applications, we propose the following device packaging: In the device configuration of Fig. 1, the weight pressing on the optical fiber may be replaced by a metal plate, which is tightened by screws to the fiber mount. The amount of signal attenuation level can be controlled by adjustment of the tightening force by screws. Grating period $\Lambda$ can be controlled easily by a small fixture on the fiber mount that adjusts the length of the spring.

Although temperature control was done in the present experiment by use of a hot plate, a small heater can be installed in the fiber mount and, if desired, a cooling plate can be added underneath the fiber mount. This combination with a temperature sensor attached to the fiber mount may permit automatic computer control.

We thank W. D. L. Finn, Faculty of Engineering, Kagawa University, for his careful proofreading of the manuscript. We also thank the reviewers for their valuable suggestions for revising this manuscript.

\section{References}

1. A. M. Vengsarkar, P. J. Lemaire, J. B. Judkins, V. Bhatia, T. Erdogan, and J. E. Sipe, "Long-period fiber gratings as bandrejection filters," J. Lightwave Technol. 14, 58-65 (1996).

2. B. H. Kim, T.-J. Ahn, D. Y. Kim, B. H. Lee, Y. Chung, U.-C. Paek, and W.-T. Han, "Effect of $\mathrm{CO}_{2}$ laser irradiation on the refractive-index change in optical fibers," Appl. Opt. 41, 3809 3815 (2002).

3. Y. J. Rao, T. Zhu, Z. L. Ran, Y. P. Wang, J. Jiang, and A. Z. Hu,
"Novel long-period fiber gratings written by high-frequency $\mathrm{CO}_{2}$ laser pulses and applications in optical fiber communication," Opt. Commun. 229, 209-221 (2004).

4. S. Savin, M. J. F. Digonnet, G. S. Kino, and H. J. Shaw, "Tunable mechanically induced long-period fiber gratings," Opt. Lett. 25, 710-712 (2000).

5. G. Rego, J. R. A. Fernandes, J. L. Santos, H. M. Salgado, and P. V. S. Marques, "New technique to mechanically induce longperiod fibre gratings," Opt. Commun. 220, 111-118 (2003).

6. D. M. Costantini, C. A. P. Muller, S. A. Vasiliev, H. G. Limberger, and R. P. Salathe, "Tunable loss filter based on metalcoated long-period fiber grating," IEEE Photon. Technol. Lett. 11, 1458-1460 (1999).

7. Y. Jiang, Q. Li, C.-H. Lin, E. Lyons, I. Tomov, and H. P. Lee, “A novel strain-induced thermally tuned long-period fiber grating fabricated on a periodic corrugated silicon fixture," IEEE Photon. Technol. Lett. 14, 941-943 (2002).

8. J. K. Bae, S. H. Kim, J. H. Kim, J. Bae, S. B. Lee, and J.-M. Jeong, "Spectral shape tunable band-rejection filter using a long-period fiber grating with divided coil heaters," IEEE Photon. Technol. Lett. 15, 407-409 (2003).

9. M. I. Braiwish, B. L. Bachim, and T. K. Gaylord, "Prototype $\mathrm{CO}_{2}$ laser-induced long-period fiber grating variable optical attenuators and optical tunable filters," Appl. Opt. 43, 17891793 (2004).

10. T. Yokouchi, Y. Suzaki, K. Nakagawa, S. Ejima, M. Yamauchi, M. Kimura, Y. Mizutani, and S. Kimura, "Fabrication and estimation of mechanically induced tunable long-period fiber grating," IEICE Trans. Electron. J88-C 1, 22-27 (2005).

11. V. Bhatia and A. M. Vengsarkar, "Optical fiber long-period grating sensors,” Opt. Lett. 21, 692-694 (1996).

12. L. Qin, Z. X. Wei, Q. Y. Wang, H. P. Li, W. Zheng, Y. S. Zhang, and D. S. Gao, "Compact temperature-compensating package for long-period fiber gratings," Opt. Mater. 14, 239-242 (2000).

13. C.-H. Lin, Q. Li, A. A. Au, Y. Jiang, E. Wu, and H. P. Lee, "Strain-induced thermally tuned long-period fiber gratings fabricated on a periodically corrugated substrate," J. Lightwave Technol. 22, 1818-1827 (2004).

14. M. N. Ng and K. S. Chiang, "Thermal effects on the transmission spectra of long-period fiber gratings," Opt. Commun. 208, 321-327 (2002). 